\title{
Radiographic findings of hemolymphangioma in four patients: A case report
}

\author{
CUI PING MAO ${ }^{1}$, YAO FENG JIN ${ }^{2}$, QUAN XIN YANG ${ }^{1}$, QIU JUAN ZHANG ${ }^{1}$ and XING HUA LI \\ Departments of ${ }^{1}$ Medical Imaging and ${ }^{2}$ Pathology, The Second Affiliated Hospital \\ of Xi'an Jiaotong University, Xi'an, Shaanxi 710004, P.R. China
}

Received April 20, 2016; Accepted February 23, 2017

DOI: $10.3892 / \mathrm{ol} .2017 .7268$

\begin{abstract}
Due to the rarity of hemolymphangioma, a limited number of cases of the disease have been reported in the literature thus far. The present case report describes the cases of 4 patients with hemolymphangioma that were diagnosed and treated at the Second Affiliated Hospital of Xi'an Jiaotong University (Xi'an, China). All patients were female, with a mean age of 44.7 years and a mean duration of symptoms prior to diagnosis of 2.7 years. The diagnosis of hemolymphangioma was determined by postoperative histopathology in all patients. A total of 2 patients were diagnosed with tumors that exhibited cystic characteristics (one in the mediastinum and the other in the neck), which was determined by computed tomography and magnetic resonance imaging. Another of the patients' tumors was located in the left forearm, and 1 patient had multifocal hemolymphangioma in the mediastinum and spleen. All patients underwent surgery and were asymptomatic during the follow-up periods (range, 8-15 months). In the present case report, the radiographic findings of the 4 cases are presented, including the unusual imaging characteristics that were observed, and relevant reports in the literature are discussed.
\end{abstract}

\section{Introduction}

Hemolymphangioma is a comparatively rare tumor comprising newly-formed lymph spaces and blood vessels (1). The tumor can occur at any age and may involve any part of the body (1-12). Hemolymphangioma typically originates from congenital malformations of the vascular and lymphatic system. Only a small number of cases have been reported in the mediastinum (13-19), spleen $(4,6)$ and neck (20). In previously reported cases, completely cystic and/or multifocal

Correspondence to: Professor Quan Xin Yang, Department of Medical Imaging, The Second Affiliated Hospital of Xi'an Jiaotong University, 157 Xiwu Road, Xi'an, Shaanxi 710004, P.R. China E-mail: quanxin1962@163.com

Key words: hemolymphangioma, mediastinum, spleen, forearm, neck hemolymphangioma were extremely rare. The correct preoperative diagnosis of hemolymphangioma remains a challenge.

The present case report describes the diagnosis, treatment and outcomes of 4 patients with hemolymphangioma that were admitted to the Second Affiliated Hospital of Xi'an Jiaotong University (Xi'an, China). All patients were female, with a mean age of 44.7 years and a mean duration of symptoms prior to diagnosis of 2.7 years. A total of 5 lesions were identified across the four cases. One patient had multifocal hemolymphangioma in the mediastinum and spleen. The other 3 patient's tumors were located in the antero-superior mediastinum, neck and left forearm, respectively. Owing to the rare nature of hemolymphangioma, preoperative diagnosis is challenging. In the present case report, the radiological findings of hemolymphangioma in 4 patients are described, including the unusual imaging characteristics observed.

\section{Case report}

Patient details. All patients were admitted to the Second Affiliated Hospital of Xi'an Jiaotong University. Written informed consent was obtained from all patients.

Case 1. A 43-year-old female patient was admitted with a complaint of a mediastinal mass identified during a medical examination $\sim 1$ year prior to admission. The patient did not complain of any other discomfort. The patient had a history of uterus teratoma detected 7 months prior to admission. No abnormalities were detected by laboratory examinations (blood routine examination and biochemical examination, including blood glucose analysis and triglyceride test). Computed tomography (CT) demonstrated a well-defined cystic mass $(5.4 \times 6.9 \times 6.6 \mathrm{~cm})$ in the left antero-superior mediastinum (Fig. 1A). The tumor was adjacent to the aortic arch and the pulmonary trunk with a homogeneous density of 15 Hounsfield units (HU) on plain CT images. Following contrast (iohexol, $350 \mathrm{mgI} / \mathrm{ml}, 4-5 \mathrm{ml} / \mathrm{s}$ ) injection, the left and anterior parts of the wall of the tumor exhibited significant enhancement, while the inside content of the mass did not exhibit any enhancement compared with pre-contrast images (Fig. 1B). Neither calcification nor enlarged lymph nodes were observed. The adjacent organs were slightly compressed and no bone destruction was observed in the neighboring spine. Based on these findings, a cystic hygroma was initially considered as the diagnosis. 
The patient underwent a tumorectomy by thoracoscopy. During the surgery, a well-defined cystic mass containing clear liquid was identified in the left side of the antero-superior mediastinum with a thin wall. Subsequently, the tumor was completely excised. Hematoxylin and eosin (H\&E) staining of the specimen was performed as previously described (5) and revealed dilated lymphatic vessels and blood vessels in the tumor (Fig. 2). A follow-up CT scan of chest revealed no recurrence $\sim 9$ months after surgery.

Case 2. A 59-year-old female patient presented with a mass on the right side of the neck. A total of $\sim 9$ months prior to presentation at the hospital, the patient identified a walnut-like mass on the right side of their neck. The mass grew gradually over the following months. At first, the patient did not feel pain or a burning sensation upon touching the mass and did not experience weight loss. As the mass grew, the patient felt slight pain/discomfort and exhibited limited cervical activity. The patient had a high blood pressure (BP) that persisted for 5 years (highest BP reading, 180/100 $\mathrm{mmHg}$ ) prior to admission and was prescribed oral medication (antihypertensive agents, taken daily) to control their BP. A total of $\sim 7$ years prior to admission, the patient underwent lobectomy of the left lobe of the thyroid for the treatment of thyroid cancer). Following the surgery, the patient took levothyroxine sodium regularly (50 $\mu \mathrm{g} /$ tablet, $1 / 4$ tablet a day).

Post-contrast CT and pre-contrast magnetic resonance imaging (MRI) were performed. The $\mathrm{CT}$ scan revealed a large cystic mass $(8 \times 6 \times 4 \mathrm{~cm})$ occupying the right side of the neck (Fig. 3). The right carotid artery and jugular vein were shifted to the left side, the right sternocleidomastoid was pressed and shifted forward, and the trachea was slightly shifted to the left. No bone destruction or enlarged lymph nodes were observed.

MRI revealed a mass lying on the right side of the neck. The mass exhibited hypo-intensity on T1-weighted images (T1WI; Fig. 4A), homogeneous hyper-intensity on T2-weighted images (T2WI; Fig. 4B) and was well defined. The inferior part of the mass was irregular (Fig. 4C). Water-fat separation MRI of the neck revealed a mass with homogeneous hypointensity on the water-suppression images (Fig. 4C) and significant hyperintensity on the fat-suppression images (Fig. 4D).

The patient underwent a surgical resection of the tumor, during which a cystic mass was observed. The diolame ruptured during surgery. The mass was completely excised and histological analysis (H\&E staining) supported a diagnosis of hemolymphangioma (Fig. 5). The patient was asymptomatic during a follow-up period of 10 months.

Case 3. A 48-year-old female patient with a known mediastinal mass detected 1 year prior to presentation, was referred for further examination and treatment due to a sporadic cough and chest pain. The patient reported slight pain on the left side of the chest which was accompanied by left shoulder pain and back pain. In addition, the patient reported eyelid weakness that became more severe when fatigued. The symptoms aggravated gradually prior to the admission. The laboratory results (blood routine examination and biochemical examination, including blood glucose analysis and triglyceride test) were all within the normal range.

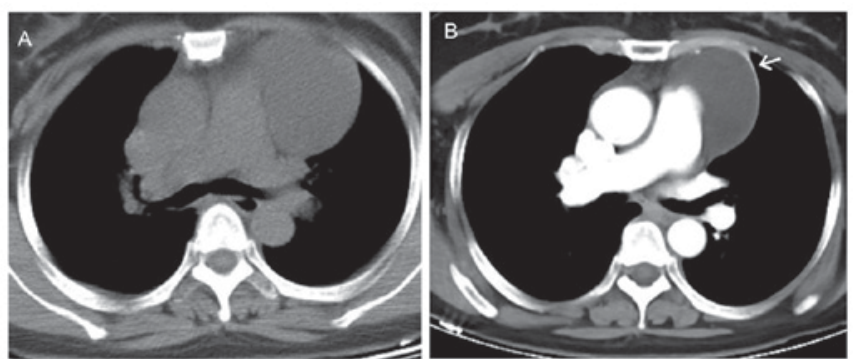

Figure 1. CT images from case 1. (A) Axial CT revealed a cystic mass with a well-defined edge within the left antero-superior mediastinum. (B) Enhanced CT identified significant enhancement for the left and anterior parts of the cyst wall (white arrow). CT, computed tomography.

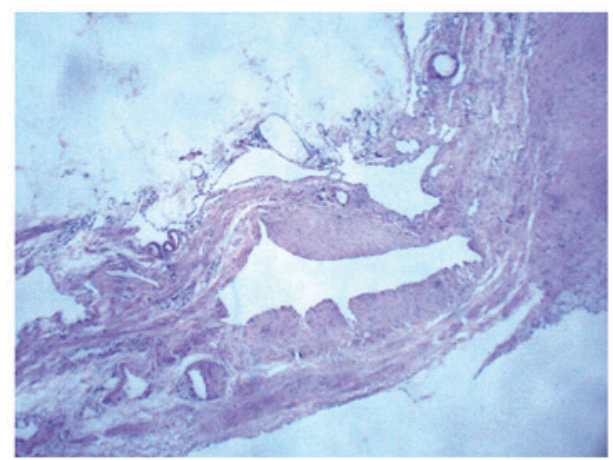

Figure 2. Histopathological section of the hemolymphangioma from case 1 with hematoxylin and eosin staining highlighting the blood and lymphatic vessels. Magnification, x100.

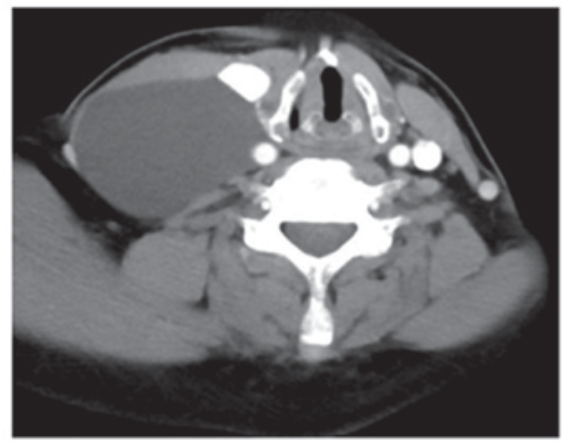

Figure 3. Imaging of case 2. Enhanced computed tomography reveals a well-defined cystic mass on the right side of the neck with no enhancement following contrast agent injection.

CT revealed a lobulated solid mass within the mediastinum, with a size of $3 \times 4 \times 5 \mathrm{~cm}$ (Fig. 6A). The tumor was adjacent to the aortic arch and left pulmonary artery. The mass showed iso-density with internal scattered calcification inside on plain CT images and progressive enhancement on post-contrast CT images (Fig. 6B and C).

Notably, the patient had a large spleen (size determined by CT, $15 \times 10 \times 10 \mathrm{~cm}$ ) and a myoma of the uterus. Multiple nodules were observed in the spleen. Progressive enhancement was observed for the majority of nodules on post-contrast (iohexol, $350 \mathrm{mgI} / \mathrm{ml}$ ) CT images (Fig. 7). These nodules were originally considered to be a hemangioma or lymphangioma. In addition, there were a small number of nodules that exhibited no enhancement, which 

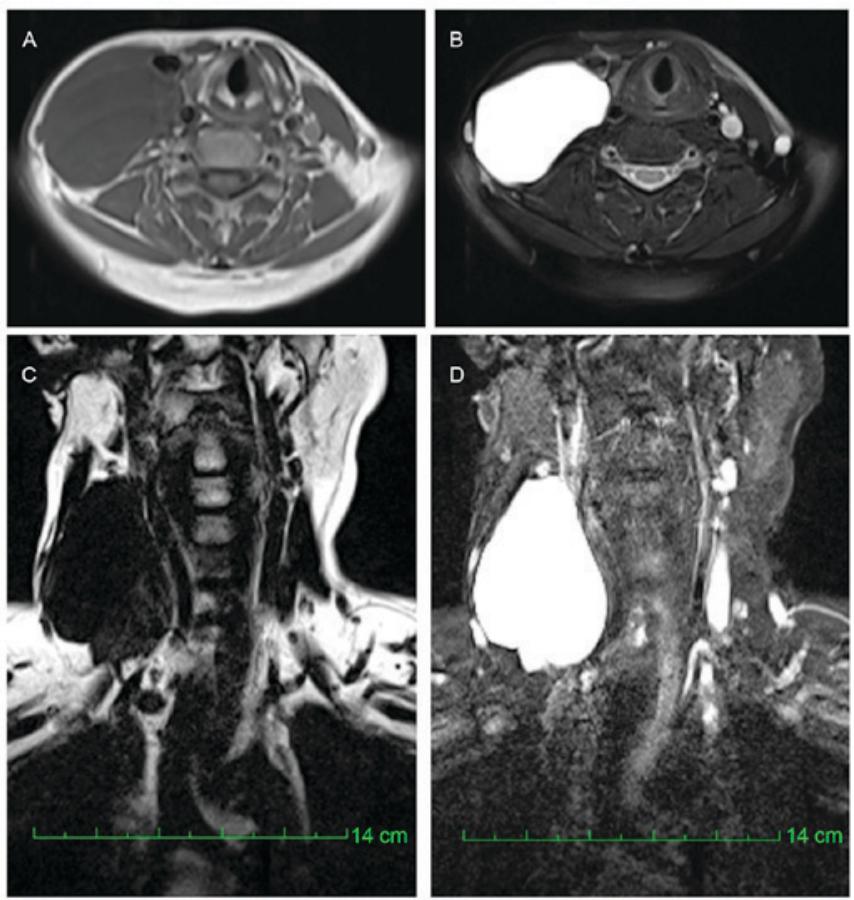

Figure 4. Magnetic resonance imaging results from case 2. A cystic mass was observed on the right side of the neck with (A) hypo-intensity on T1-weighted images (B) and hyper-intensity on T2-weighted images. The tumor demonstrated (C) hypo-intensity on the water-suppression images and (D) significant hyper-intensity on the fat-suppression images

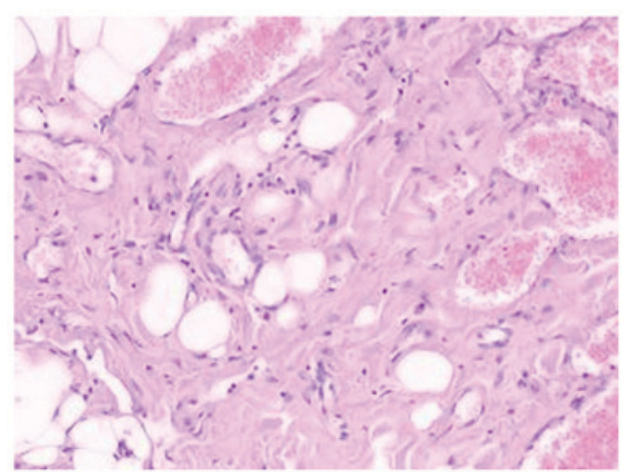

Figure 5. Histological results of case 2. Hematoxylin and eosin staining of the tissue supported a diagnosis of hemolymphangioma. Magnification, x100.

were considered to be cysts. The patient underwent resection of the mediastinal mass and a splenectomy. The interval between the two surgeries was 9 months. H\&E staining of samples from the two surgeries revealed a diagnosis of hemolymphangioma for the mediastinal mass (Fig. 8) and the spleen nodules (Fig. 9). There was no recurrence at the 15 month follow-up.

Case 4. A 29-year-old female patient was admitted to the Second Affiliated Hospital of Xi'an Jiaotong University with a mass on the left forearm that was identified $\sim 8$ years prior to admission. Upon admission, the left forearm exhibited markedly more swelling compared with its contra-lateral counterpart, with pain upon pressing. The laboratory findings were normal (blood routine examination and biochemical examination, including blood glucose analysis and triglyceride test). An osteosarcoma was initially considered as a diagnosis.
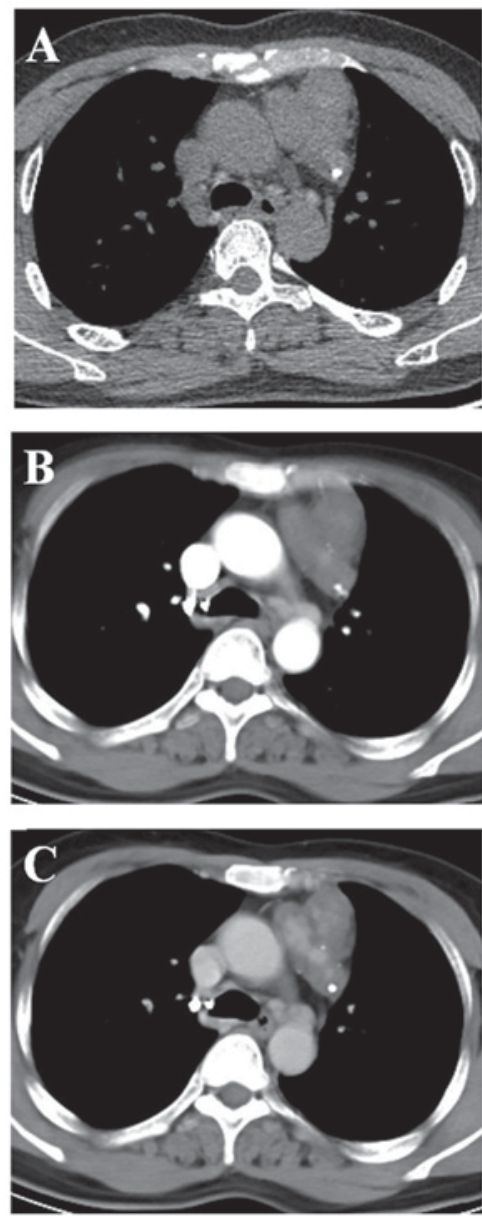

Figure 6. CT images from case 3. (A) A lobulated tumor was observed within the mediastinum. The tumor exhibited iso-density with internal scattered calcification. A progressive enhancement of the tumor was observed on post-contrast CT images: (B) Arterial phase and (C) venous phase. The enhancement is stronger in the venous phase compared with the arterial phase.

MRI revealed an irregular mass on the left forearm, with heterogeneous intensities on T1WI (Fig. 10A) and hyper-intensity on T2WI (Fig. 10B and C). On fat-suppression T2WI, multiple small round-like hypo-intensity was observed inside the tumor (Fig. 10B and C). A diagnosis of a malignant osteosarcoma with infiltration of the left ulna was initially considered. The mass was resected and a grayish-yellow mass was identified in the lateral side of the proximal left forearm with a size of $2 \times 5 \times 8 \mathrm{~cm}$. Histopathological features from $\mathrm{H} \& \mathrm{E}$ staining of the mass revealed a hemolymphangioma (Fig. 11). The postoperative course was uneventful. The patient was asymptomatic during a follow-up period of 8 months.

\section{Discussion}

Hemolymphangioma is a rare tumor composed of cystically dilated lymphatic and blood vessels (4). The pathogenesis of this disease remains unclear. In the majority of cases, the tumor is considered to be a congenital malformation of the vascular system. The obstruction of the venolymphatic communication between dysembrioplastic vascular tissue and the systemic circulation may contribute to the formation of hemolymphangioma (21). Lymphatic vessel injury as a result of trauma or surgery results in inadequate lymph fluid drainage, 


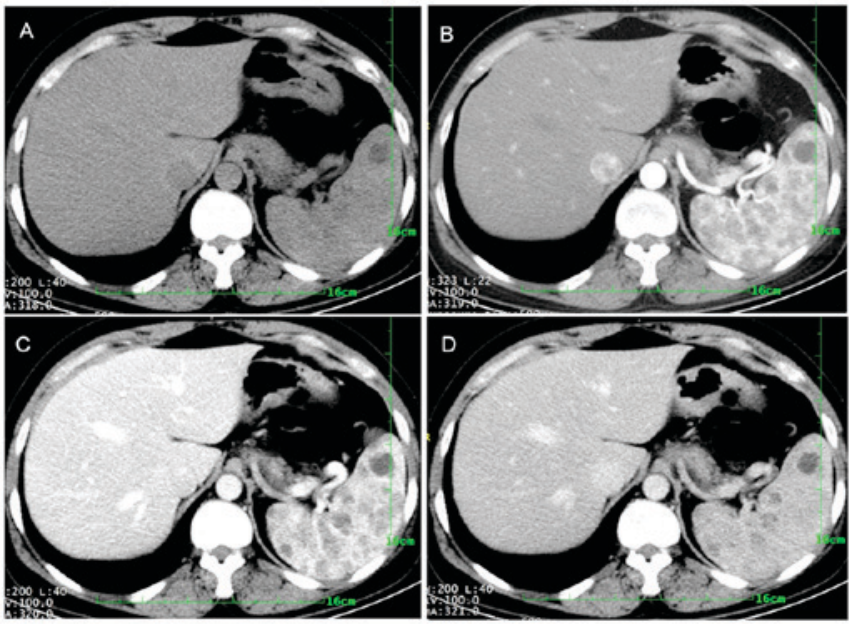

Figure 7. CT images from case 3. (A) CT imaging revealed multiple low-density nodules in the spleen. (B) Arterial phase following contrast injection, (C) portal venous phase and (D) delayed phase. Progressive enhancement was observed for the majority of the nodules. The enhancement is gradually intensified from arterial phase to delayed phase following contrast injection. There were a number of nodules that exhibited no enhancement, which were considered to be cysts. CT, computed tomography.

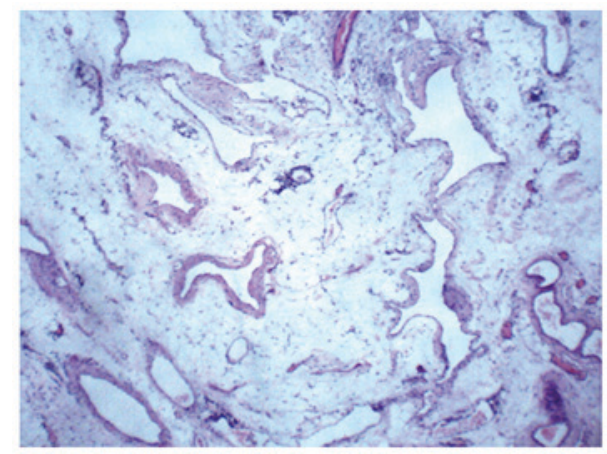

Figure 8. Hematoxylin and eosin staining of the mediastinal tumor from case 3 indicated a diagnosis of hemolymphangioma. Magnification, x100.

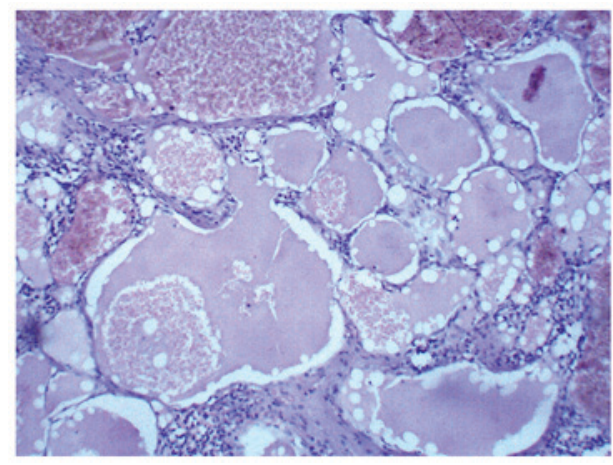

Figure 9. Histopathological analysis (hematoxylin and eosin staining) of the spleen from case 3 suggested a diagnosis of hemolymphangioma. Magnification, $\mathrm{x} 200$.

which is regarded as another cause of hemolymphangioma (8). In the present study, five hemolymphangioma lesions across 4 patients are reported. One of the patients had a history of thyroid surgery, which may have contributed to the formation of the tumor. The majority of patients with hemolymphangioma
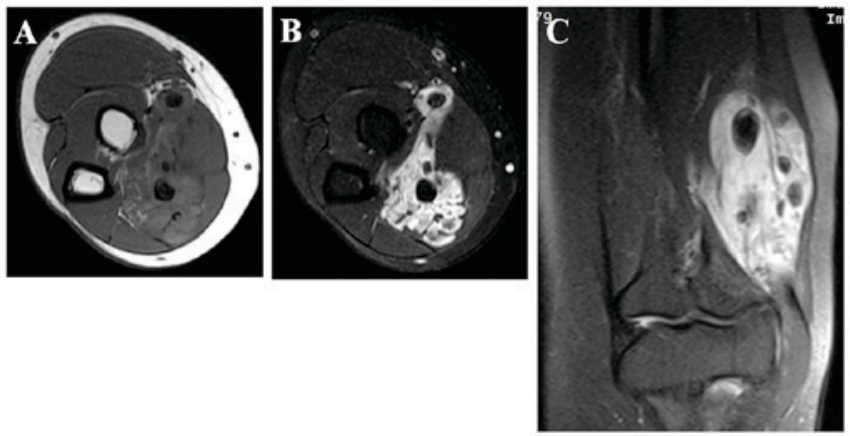

Figure 10. Magnetic resonance imaging of case 4. (A) T1WI revealed a tumor with heterogeneous intensities compared with the surrounding muscles. (B and C) The tumor demonstrated hyper-intensity with scattered round-like low intensity on T2WIs following fat-suppression. WI, weighted image.

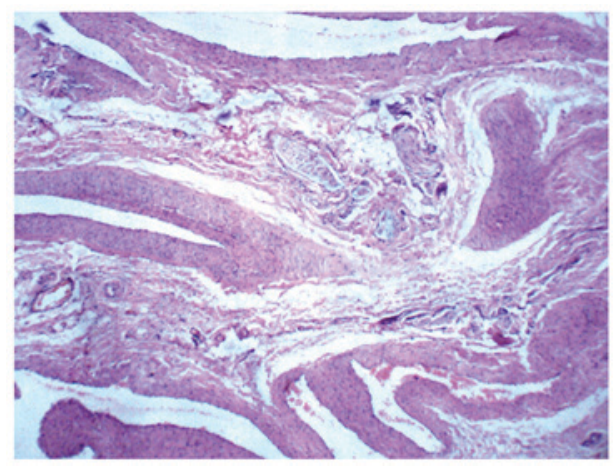

Figure 11. Hematoxylin and eosin staining of case 4 supported a diagnosis of hemolymphangioma. Magnification, x100.

are asymptomatic for a long period of time and the discomfort typically originates from the tumor as it grows (22).

Hemolymphangioma can be located at any site of the body, including the pancreas $(1,7,10,21,23-28)$, spleen $(4,6)$, waist (5), duodenum (29), small intestine (30), rectum $(8,12)$, gastrointestinal system (31), chest wall (9), mediastinum (13-18), extremities $(22,32,33)$, orbit (34-36), esophagus (37), mouth $(38,39)$, neck (20), retroperitoneum (40), vertebrae (2), spermatic cord (41), knee (42), colon (43), vulva (44) and testis (45). Nevertheless, multi-focal hemolymphangioma is rare. Only one previous study has reported on a female patient with hemolymphangioma in the spleen and retroperitoneum (3). Of the 4 cases in the present case report, there was 1 patient who had multifocal hemolymphangioma in the mediastinum and spleen. This made a differential diagnosis challenging.

The clinical diagnosis of hemolymphangioma is not a common occurrence, due to its rarity and the absence of distinctive symptoms. CT and MRI are useful in defining the extent and the invasion of the tumor, and planning the surgical strategy. Hemolymphangioma typically presents as a cystic-solid or solid tumor. The cystic part may be caused by the rupture and fusion of the vascular cavity, and the solid part may represent the residual and compressed vascular tissue (7). The imaging characteristics depend on the composition of blood vessels and any accompanying infection or bleeding.

Different sizes of blood vessels in the hemolymphangioma may produce different enhanced characteristics upon imaging (1). Significant and persistent enhancement can be 
observed in tumors rich in blood vessels and a septum may show marked enhancement. In the current case report, the lesions in the mediastinum and spleen in case 3 exhibited progressive enhancement. The imaging findings were similar to cavernous hemangioma. In case 1 , the enhanced cyst wall suggested that the tumor contained a significant blood vessel component. Completely cystic hemolymphangioma has rarely been reported previously $(13,14,46)$. One distinctive radiographic finding from the present case report is that the tumors in the first two cases were similar, with cystic characteristics. In particular, no enhancement was observed on post-contrast CT images for case 2, which made the diagnosis more challenging.

MRI can aid in determining the association between hemolymphangioma and the surrounding tissues, and the extent of invasion. The tumor in case 4 exhibited heterogeneous iso-intensity on T1WI and hyper-intensity on T2WI, indicating the presence of a lower number of tortuous blood vessels and water-based substances in the lesions. CT and MRI can aid in the selection of surgical strategy and follow-up treatment. However, a definitive diagnosis should be based on histological evidence.

The cystic-solid and cystic lesions should be taken into account during the differential diagnosis of hemolymphangioma, including lymphangiomas and thymus cysts. Lymphangioma is a rare, benign lesion derived from a malformation of the lymphatic system, which is frequently identified in the head, neck and axilla. Lymphangioma is commonly diagnosed in children $<2$ years old, although it may occur at any age (15). Lymphangiomas are typically composed of cystically dilated lymphatic vessels (47). It is challenging to differentiate between cystic hemolymphangioma and cystic lymphangioma by imaging alone, although enhanced CT may aid in distinguishing them. Cystic lymphangioma is common and is typically observed in infants $(47,48)$. Combining imaging techniques with clinical symptoms and pathologic findings may aid in determining the correct diagnosis.

A cystic mediastinal hemolymphangioma should be differentiated from a thymus cyst. A thymus cyst is typically located in the neck and supra anterior mediastinum, and there are typically no clinical symptoms. The flowing void effect of the vessel component inside the cyst in case 3 from the present case report may aid with the diagnosis of hemolymphangioma.

The majority of cases of hemolymphangioma are diagnosed in female patients. A recent literature review reported that $>2 / 3$ $(15 / 22)$ reported cases of hemolymphangioma were in female patients. In the present study, the patients were all female. However, no direct evidence has been reported for the gender-associated dominance of hemolymphangioma, to the best of our knowledge.

Although hemolymphangioma is typically a benign disease, the recurrence and invasion of adjacent organs has been reported (24). Complete surgical resection is considered to be the most effective treatment of the hemophymphangioma $(22,29)$; however, this needs to be performed carefully in order to avoid possible haemorrhage due to the vascular component of hemolymphangioma. Other treatment options include sclerotherapy, electrocautery, radium implantation, cyrosurgery and laser therapy. Angiography and embolization can also be performed in cases of acute bleeding (30). The aim of treatment is to remove the entire tumor. The majority of cases in the literature had successful postoperative courses of treatment. Postoperative follow-up is necessary due to the potential recurrence or metastasis of the tumor (3). All cases in the present case reports remained asymptomatic during postoperative follow-up period.

In conclusion, the current case report describes five hemolymphangioma lesions in 4 female patients. Completely cystic and multifocal masses are rare. An accurate diagnosis cannot typically be established preoperatively despite modern imaging techniques. The possibility of a hemolymphangioma should be considered when cystic and cystic-solid masses occur.

\section{References}

1. Pan L, Jian-bo G and Javier PT: CT findings and clinical features of pancreatic hemolymphangioma: A case report and review of the literature. Medicine 94: e437, 2015.

2. Zhang Y, Li B, Shi H, Cai L and Hou J: Vertebral hemangiolymphangioma mimics bone metastases on $99 \mathrm{mTc}-\mathrm{MDP}$ SPECT/CT. Clin Nucl Med 41: 76-78, 2016.

3. Zhang DY, Lu Z, Ma X, Wang QY, Sun WL, Wu W and Cui PY: Multiple hemolymphangioma of the visceral organs: A case report and review of the literature. Medicine 94: e1126, 2015.

4. Mei Y, Peng CJ, Chen L, Li XX, Li WN, Shu DJ and Xie WT: Hemolymphangioma of the spleen: A report of a rare case. World J Gastroenterol 21: 5442-5444, 2015.

5. Li Y, Pang X, Yang H, Gao C and Peng B: Hemolymphangioma of the waist: A case report and review of the literature. Oncol Lett 9: 2629-2632, 2015.

6. Zhang Y, Chen XM, Sun DL and Yang C: Treatment of hemolymphangioma of the spleen by laparoscopic partial splenectomy: A case report. World J Surg Oncol 12: 60, 2014.

7. Dong F, Zheng $\mathrm{Y}$, Wu JJ, Fu YB, Jin $\mathrm{K}$ and Chao $\mathrm{M}$ : Hemolymphangioma: A rare differential diagnosis of cystic-solid or cystic tumors of the pancreas. World J Gastroenterol 19: 3520-3523, 2013

8. Chen G, Cui W, Ji XQ and Du JF: Diffuse hemolymphangioma of the rectum: A report of a rare case. World J Gastroenterol 19: 1494-1497, 2013.

9. Zhang $X$, Sheng $X$, Liu F, Jian R, Li L and Luo R: Hemolymphangioma of the chest wall: A rare case report. Oncol Lett 3: 816-818, 2012.

10. Balderramo DC, Di Tada C, de Ditter AB and Mondino JC: Hemolymphangioma of the pancreas: Case report and review of the literature. Pancreas 27: 197-199, 2003.

11. Pandey S, Fan M, Chang D, Zhu J, Zhu Y and Li Z: Hemolymphangioma of greater omentum: A rare case report. Medicine 95: e3508, 2016.

12. Pandey S, Fan M, Zhu J, Lu X, Chang D and Li X: Unusual cause of 55 years of rectal bleeding: Hemolymphangioma (a case report). Medicine 96: e6264, 2017.

13. Zehani A, Ayadi-Kaddour A, Cherif J, Marghli A, Beji M, Kilani T and El Mezni F: Cystic mediastinal hemolymphangioma. Tunis Med 90: 754-755, 2012 (In French).

14. Bosdure E, Mates M, Mely L, Guys JM, Devred P and Dubus JC: Cystic intrathoracic hemolymphangioma: A rare differential diagnosis of acute bronchiolitis in an infant. Arch Pediatr 12: 168-172, 2005 (In French).

15. Riquet M, Briere J, Le Pimpec-Barthes F, Bely N, Dujon A, Velly JF, Brichon PY, Faillon JM, Mouroux J, Jancovici R and Dahan M: Cystic lymphangioma of the neck and mediastinum: Are there acquired forms? Report of 37 cases. Rev Mal Respir 16: 71-79, 1999.

16. NatafP, Mestiri T, Martin de Lasalle E, Benomar M, Gandjbakhch I and Cabrol C: Pericardial hemolymphangioma. Apropos of a case. Arch Mal Coeur Vaiss 81: 1137-1140, 1988 (In French).

17. Contamin C, Denis B, Mallion JM, Rival MA, Martin-Noel P, Latreille R and Barrie J: Heart hemolymphangioma. Apropos of a case. Coeur Med Interne 12: 671-678, 1973.

18. Bagolan P, Alati E and Fisicaro M: Some defects of development of the mediastinum; three cases: One cavernous hemolymphangioma and two cystic hygromas. Archivio Chir Torace 10: 559-573, 1953 (In undetermined language).

19. Wu J, Shangguan H, Zhou S and Dong L: Hemolymphangioma in the posterior mediastinum: A case report and literature review. Clin Respir J: Mar 7, 2016 (Epub ahead of print). 
20. Gaillard de Collogny L and Delage J: Cervical hemolymphangioma in a young patient. J Fr Otorhinolaryngol Audiophonol Chir Maxillofac 30: 469-473, 1981.

21. Figueroa RM, Lopez GJ, Servin TE, Esquinca MH and Gómez-Pedraza A: Pancreatic hemolymphangioma. JOP 15: 399-402, 2014

22. Kosmidis I, Vlachou M, Koutroufinis A and Filiopoulos K: Hemolymphangioma of the lower extremities in children: Two case reports. J Orthop Surg Res 5: 56, 2010.

23. Sun LF, Ye HL, Zhou QY, Ding KF, Qiu PL, Deng YC, Zhang SZ and Zheng S: A giant hemolymphangioma of the pancreas in a 20-year-old girl: A report of one case and review of the literature. World J Surg Oncol 7: 31, 2009.

24. Toyoki Y,Hakamada K, Narumi S, Nara M, Kudoh D, Ishido K and Sasaki M: A case of invasive hemolymphangioma of the pancreas. World J Gastroenterol 14: 2932-2944, 2008.

25. Banchini E, Bonati L and Villani LG: A case of hemolymphangioma of the pancreas. Minerva Chir 42: 807-813, 1987 (In Italian).

26. Montete P, Marmuse JP, Claude R and Charleux $\mathrm{H}$ : Hemolymphangioma of the pancreas. J Chir (Paris) 122: 659-663, 1985 (In French).

27. Couinaud C, Jouan, Prot, Chalut, Favre and Schneiter: A rare tumor of the head of the pancreas. (Hemolymphangioma weighing 1,500 kg.). Presse Med 75: 1955-1956, 1967 (In French)

28. Couinaud, Jouan, Prot, Chalut and Schneiter:Hemolymphangioma of the head of the pancreas. Mem Acad Chir (Paris) 92: 152-155, 1966 (In French).

29. Antonino A, Gragnano E, Sangiuliano N, Rosato A, Maglio M and De Palma M: A very rare case of duodenal hemolymphangioma presenting with iron deficiency anemia. Int J Surg Case Rep 5: 118-121, 2014

30. Fang YF, Qiu LF, Du Y, Jiang ZN and Gao M: Small intestinal hemolymphangioma with bleeding: A case report. World J Gastroenterol 18: 2145-2146, 2012.

31. Kim WT, Lee S and Lee JU: Bleeding gastric hemolymphangioma: Endoscopic therapy is feasible. Dig Endosc 25: 553-554, 2013.

32. Sideri V, Zavras N, Efi T, Niktari G, Kyrkou I, Kanouras G, MexiBourna $\mathrm{P}$ and Fretzayas A: A hemolymphangioma in the arm and the hand of a neonate. Early Human Dev 86: S104-S105, 2010.

33. Cole DJ, Sood SC and Broomhead IW: Pulmonary embolism associated with hemolymphangioma of lower extremity. Plast Reconstr Surg 63: 265-268, 1979.

34. Chanfi M: Hemolymphangioma of the orbit in a young girl: A clinical observation. J Fr Ophtalmol 27: 1047-1049, 2004.

35. Ben Chehida F, Chahed N, Turki H, Hammou Jeddi A, Zitouna MM, Bettaieb A and Gharbi HA: Hemolymphangioma of the orbit in children. Apropos of a case. Ann Radiol (Paris) 28: 626-628, 1985.
36. Guillot M, Dufier JL, Pierre-Kahn A, Nihoul-Fekete C, Lenoir G and Haye C: Hemolymphangioma of the orbit in children. Arch Fr Pediatr 40: 401-403, 1983 (In French).

37. Canavese F, Cortese MG, Proietti L, Costantino S, Rosina M, Nangeroni M, Defilippi C and Di Rosa GP: Bulky-pedunculated hemolymphangioma of the esophagus: Rare case in a two-years old girl. Eur J Pediatr Surg 6: 170-172, 1996.

38. Bureau Y, Delaire J, Barrière H, Litoux P and Bureau B: Hemolymphangioma of the tongue. Results of surgical treatment. Bull Soc Fr Dermatol Syphiligr 73: 422-423, 1966 (In French).

39. Ullik R: On a hemolymphangioma of the floor of the mouth Wien Klin Wochenschr 71: 958-960, 1959 (In German).

40. Kanaitsuka T, Itani K, Shigeta H, Yamamura Y, Kogawa T, Yoshikawa T, Sugino S, Kanatsuna T, Kondou M, Takashina K, et al: A case report of giant retroperitoneal hemolymphangioma. Nihon Naika Gakkai Zasshi 76: 1595-1603, 1987.

41. Rogel-Rodríguez JF, Gil-García JF, Velasco-García P, RomeroEspinoza F, Zaragoza-Salas T and Muñoz-Lumbreras G: Hemangiolymphangioma of the spermatic cord in a 17 year-old: A case report. Cir Cir 84: 164-168, 2016.

42. ParraBA,ValenciaZN,Espinal BD and MayaAI:Low-flow synovial vascular malformation of the knee (hemangiolymphangioma)Case report. Rev Chil Pediatr 86: 43-46, 2015 (In Spanish).

43. Castro-Poças F, Lobo L, Amaro T, Soares J and Saraiva MM Colon hemangiolymphangioma-a rare case of subepithelial polyp. Int J Colorectal Dis 30: 989-990, 2015.

44. Sapountzis S, Singhal D and Chen HC: Radical resection and reconstruction with bilateral gluteal fold perforator flaps for vulvar hemangiolymphangioma. Int J Gynaecol Obstet 121: 179-180, 2013.

45. Shin YS, Doo AR, Kim MK, Jeong YB and Kim HJ: Cavernous hemangiolymphangioma of the testis without cutaneous hemangiomatosis in an elderly patient. Korean J Urol 53: 810-812, 2012.

46. Gossot D, Decazes JM, Sarfati E and Dubost C: Cystic hemolymphangioma of the adrenal gland. J Chir (Paris) 124: 404-405, 1987 (In French).

47. DI Marco M, Grassi E, Vecchiarelli S, Durante S, Macchini M and Biasco G: Retroperitoneal lymphangioma: A report of 2 cases and a review of the literature regarding the differential diagnoses of retroperitoneal cystic masses. Oncol Lett 11: 3161-3166, 2016

48. Yang B, Jiang C, Zhang B, Ren Q, Tang T, Xu S, Xu H, Yao H, Han Y, Liu S, et al: Giant primary cystic mediastinal lymphangioma: A case report. Oncol Lett 8: 1246-1248, 2014.

This work is licensed under a Creative Commons Attribution-NonCommercial-NoDerivatives 4.0 International (CC BY-NC-ND 4.0) License. 